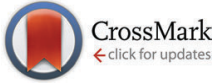

Cite this: Phys. Chem. Chem. Phys., 2015, 17, 29150

Received 27th August 2015, Accepted 7th October 2015

DOI: $10.1039 / c 5 c p 05124 j$

www.rsc.org/pccp

\title{
Direct observation of epitaxial organic film growth: temperature-dependent growth mechanisms and metastability $\dagger$
}

\author{
Helder Marchetto, ${ }^{\mathrm{ab}}$ Thomas Schmidt, ${ }^{\text {ac }}$ Ullrich Groh, ${ }^{\mathrm{c}}$ Florian C. Maier, ${ }^{\mathrm{cd}}$ \\ Pierre L. Lévesque, ${ }^{\text {ae }}$ Rainer H. Fink, ${ }^{f}$ Hans-Joachim Freund ${ }^{a}$ and Eberhard Umbach ${ }^{\text {ac }}$
}

\begin{abstract}
The growth of the first ten layers of organic thin films on a smooth metallic substrate has been investigated in real-time using the model system PTCDA on Ag(111). The complex behaviour is comprehensively studied by electron microscopy, spectroscopy and diffraction in a combined PEEM/LEEM instrument revealing several new phenomena and yielding a consistent picture of this layer growth. PTCDA grows above room temperature in a Stranski-Krastanov mode, forming three-dimensional islands on a stable bi-layer, in competition with metastable 3rd and 4th layers. Around room temperature this growth mode changes into a quasi layer-by-layer growth, while at temperatures below about $250 \mathrm{~K}$ a Vollmer-Weber-like behaviour is observed. By means of laterally resolved soft X-ray absorption spectroscopy the orientation of all adsorbed molecules is found to be homogeneously flat lying on the surface, even during the growth process. The films grow epitaxially, showing long-range order with rotational domains. For the monolayer these domains could be directly analysed, showing an average size of several micrometers extending over substrate steps.
\end{abstract}

\section{Introduction}

Organic thin films have gained enormous interest in recent years because of the large variety of systems with tailored properties, many open scientific questions, and the increasing importance of organic electronic devices like light emitting diodes, solar cells, field-effect transistors, etc. ${ }^{1-3}$ It is established that in general the optical and electronic properties of such films may strongly depend on their structural properties. ${ }^{4}$ These, in turn, are not only influenced by the molecular shape and the intermolecular interaction forces but also by the preparation conditions including parameters like type of substrate, chemical and geometric structure of the substrate surface, geometry and functional groups of the molecules, deposition rate and temperature. Thus, for the optimization of function and efficiency of

\footnotetext{
${ }^{a}$ Fritz-Haber-Institut der Max-Planck-Gesellschaft, Chemische Physik, 14195 Berlin, Germany. E-mail: schmidtt@fhi-berlin.mpg.de

${ }^{b}$ ELMITEC Elektronenmikroskopie GmbH, Albrecht-von-Groddeck-Str.3, 38678 Clausthal-Zellerfeld, Germany

${ }^{c}$ Universität Würzburg, Experimentelle Physik, Am Hubland, 97074 Würzburg, Germany

${ }^{d}$ Forschungszentrum Jülich GmbH, IEK-5 Photovoltaik, 52425 Jülich, Germany

${ }^{e}$ Université de Montréal, Département de Chimie, Montréal, Québec H3C 3J7, Canada

${ }^{f}$ Friedrich-Alexander-Universität Erlangen-Nürnberg, Department Chemie und Pharmazie, 91058 Erlangen, Germany

$\dagger$ Electronic supplementary information (ESI) available. See DOI: 10.1039/ c5cp05124j
}

an organic thin film a deep understanding of the mechanisms of film growth and interface formation is required. ${ }^{1-4}$ Compared to the very well understood atomic growth of metals or inorganic semiconductors, the growth of organic molecular films tends to be much more complex due to the larger size of the molecules, different bonding mechanisms, role of functional groups, anisotropic molecular structure, molecular orientation, etc. ${ }^{5}$

An ideal instrument to study many of these properties and their influence on the growth is the spectro-microscope SMART (Spectro-Microscope with Aberration correction for many Relevant Techniques), ${ }^{6-8}$ which combines photoemission (UV-light or X-ray induced, i.e. UV-PEEM or XPEEM) with a low-energy electron microscope (LEEM) comprising an imaging energy analyzer and a unique aberration correction system for high spectroscopic resolution, high spatial resolution, and high sensitivity. This instrument enables in situ and real-time experiments for the investigation of, e.g., the growth mode, its temperature dependence, the influence of substrate morphology, the internal structure of deposited nano-objects, and several other properties.

The model system PTCDA on Ag(111) is one of the most studied organic film systems since it is rather stable, can very accurately and purely be prepared, and can even be tuned for epitaxial growth. PTCDA, a planar molecule with $D_{2 \mathrm{~h}}$ symmetry (see inset in Fig. 1a), has mainly been investigated by integral and therefore laterally averaging methods like electron spectroscopies, ${ }^{9}$ high-resolution electron energy loss spectroscopy, ${ }^{10}$ X-ray standing waves, ${ }^{11}$ or diffraction methods, ${ }^{12,13}$ but also 


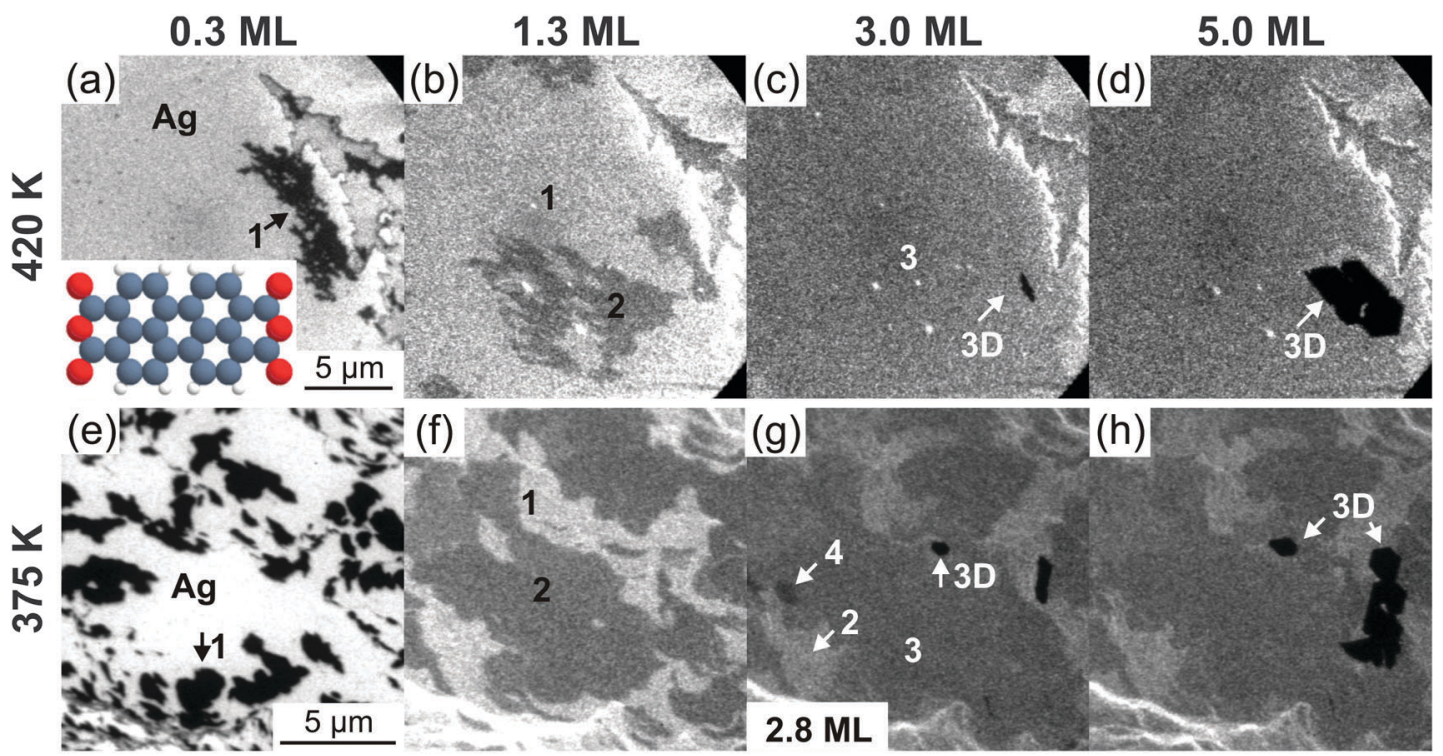

Fig. 1 Growth of PTCDA/Ag(111) observed at $420 \mathrm{~K}$ (upper row) and $375 \mathrm{~K}$ (lower row), respectively, by UV-PEEM images taken during growth; deposition rate $0.2 \mathrm{ML}$ per min. The columns indicate the coverage, the labels $\mathrm{Ag}, 1$ to 4 , and $3 \mathrm{D}$ are meant to help the identification of the Ag substrate, the 1st to the 4th PTCDA layer, and the three-dimensional islands. The detector amplification was adjusted between some of the figures in order to optimize the contrast, since attenuation causes an exponential decrease of the intensity. Therefore, one cannot use a common grayscale. The PTCDA molecule is depicted in the inset of (a) with carbon, oxygen and hydrogen atoms in black, red and white colours.

local probe (e.g. STM) experiments have been performed, especially in the (sub)mono- and bilayer regime. ${ }^{14-17}$ Very recently, some co-deposition studies adding similar molecules (e.g., Cu-Pc) to PTCDA/Ag(111) also appeared in the literature (see, e.g. ref. 18). The model system has also been proven useful for phase reconstruction from a focus series in LEEM experiments. ${ }^{19}$

Whereas above room temperature PTCDA apparently grows in a Stranski-Krastanov mode, a quasi layer-by-layer growth has been derived ${ }^{13}$ from diffraction data taken below $350 \mathrm{~K}$ and is directly observed and proven here. The growth is not only determined by the substrate temperature (and deposition rate), but also by the substrate morphology which influences the nucleation process. ${ }^{20}$ The start of the growth process, i.e. the interaction of the first organic layer at the interface to the substrate may play a decisive role for the growth of the entire film and hence also for its electronic and optical properties. In the case of PTCDA on Ag the first layer is chemisorbed and hence bonds more strongly than the subsequent layers. ${ }^{9}$ The monolayer may form a commensurate superstructure with long-range order, ${ }^{13,14,17,21}$ consisting of several (rotational and mirror) domains. NEXAFS investigations of the monolayer and of thick films revealed that the PTCDA molecules are oriented flat-lying, i.e. parallel to the surface. ${ }^{9,22}$ Because of their integral character, most of the experiments were insensitive to low concentrations of inhomogeneities like, e.g., 3D islands or defects which may play an important role for the electronic and optical properties.

The SMART microscope with several different operation modes (see Section 2.3) enables new and deeper insights into organic layer growth. The possibility of direct and real-time observation (Sections 3.1-3.3) allows the monitoring of all stages of growth and facilitates a reproducible preparation of specific film structures and thicknesses. Laterally resolved NEXAFS spectroscopy (Section 3.4) is highly sensitive to chemical and orientational inhomogeneities, even if they cover only a small surface portion (e.g. less than $0.1 \%$, which is much less than the detection limit of differences in integral measurements). The linear dichroism contrast is used to investigate the molecular orientation during the growth process (Section 3.4). Finally, the structural sensitivity of back-diffracted low-energy electrons is applied to determine the geometrical structure, orientation, size of the rotational domains of the monolayer and their interaction with substrate atomic steps (Section 3.5). We finally note that only very few studies using PEEM or LEEM instruments for the investigation of organic thin films exist in the literature, and these studies concern different molecules (see, e.g., ref. 23) or only certain aspects (see, e.g., ref. 18).

\section{Experimental}

The SMART spectro-microscope used for the experiments has been built within a collaboration of several German groups from the universities Würzburg, Erlangen, Clausthal, the Fritz-Haber Institute of the Max-Planck Society, the Zeiss company, and BESSY (now HZB).$^{6-8}$ The microscope is installed at the BESSY-II storage ring of the Helmholtz-Center Berlin for Material and Energy (HZB).

\subsection{Instrumental set-up}

SMART is a new type of spectro-microscope that combines a "standard" LEEM/PEEM instrument with aberration corrections (both, chromatic and spherical aberrations) ${ }^{40-43}$ and with energy resolution using an imaging energy filter. Theoretically, 
a lateral resolution of $0.5 \mathrm{~nm}$ at an energy resolution of less than $100 \mathrm{meV}$ is achievable at nearly 60 times higher transmission compared to conventional LEEM/PEEM instruments due to a large acceptance angle which can be used because of the aberration corrections. Experimentally demonstrated is a (conservatively measured) lateral resolution of $2.6 \mathrm{~nm}$ in LEEM and $18 \mathrm{~nm}$ in energy-filtered XPEEM, ${ }^{42,43}$ respectively, and an increase in transmission by a factor of $6 .{ }^{43}$ The instrument is installed at a soft X-ray, high flux-density undulator beam line at BESSY II with tunable photon energy range between 100 and $1000 \mathrm{eV}$ and high photon energy resolution $(<0.1 \mathrm{eV})$. Additionally, SMART is equipped with a $\mathrm{Hg}$ short arc lamp as UV light source $(h \nu=4.9 \mathrm{eV})$ and an electron gun. The special so-called Apple type undulator source ${ }^{34,35}$ allows to vary the direction of the linear polarization or the helicity of the circular polarization of the X-rays. The design of the specimen chamber enables the deposition of, e.g., organic material under grazing incidence $\left(20^{\circ}\right)$ on the sample surface at the measurement position in front of the objective lens. Therefore, real time observations of the growth could be performed. The experiments have been carried out with two versions of the SMARTmicroscope: (i) an intermediate PEEM set-up with energy filter but without aberration correction and (ii) the final complete set-up with aberration correction and electron gun.

\subsection{Sample and film preparation}

A Ag(111) single crystal oriented within an accuracy better than $0.2^{\circ}$ has been cleaned by cycles of Ar-sputtering (600 eV, $1 \mu \mathrm{A}$, $5 \times 10^{-5} \mathrm{mbar}, 15 \mathrm{~min}$, room temperature) with subsequent annealing at $700-800 \mathrm{~K}$ for about $15 \mathrm{~min}$. The cleaning progress was checked by PEEM, LEEM, LEED, and XPS. After several PTCDA adsorption and cleaning cycles the crystal surface developed into an alternating sequence of about $10 \mu \mathrm{m}$ wide flat areas with atomic step distances of up to several $100 \mathrm{~nm}$ and of rough areas with a high concentration of steps and step bunches.

For the in situ preparation of the PTCDA films a Knudsen cell type evaporator was used. The deposition rate was set to about 0.2 ML per min and 0.08 ML per $\min (\mathrm{ML}=$ monolayer coverage) in the real-time PEEM and in the NEXAFS-PEEM experiments, respectively. $1 \mathrm{ML}$ corresponds to the deposited amount required to saturate the first PTCDA layer (of parallel oriented molecules) on the surface at $330 \mathrm{~K}$ (at this temperature desorption can be neglected). The base pressure of the measurement chamber was $<3 \times 10^{-10}$ mbar.

A potential influence of the instrumental set-up on the growth has been carefully checked and could be excluded by "blind" experiments, where no accelerating electric field, no UV-light, or no electrons were applied to the sample. For this purpose the growth process, for instance, was recorded by rare snap shots, where every $2 \mathrm{~min}$ high voltage and light were activated for only about $5 \mathrm{~s}$. Since in this case we found no changes of the growth behaviour with respect to the situation with high voltage and UV light continuously applied, we are sure that electric field and UV light have no influence on the growth. However, for high flux densities of electrons or X-rays exposuredependent beam damage could be observed, for instance by a reduction of the respective signals. Therefore, to avoid beam damage the flux density was reduced, the acquisition time and process speed optimized, and the probed field of view chosen large enough to increase the signal-to-noise ratio in the images. Additionally, in LEEM experiments the kinetic energy of the electron beam at the sample surface was reduced below $5 \mathrm{eV}$ which is about the threshold below which no beam damage occurred.

\subsection{Multi-method approach for comprehensive characterization}

The complexity of the organic growth requires a comprehensive characterization. The above mentioned observations, namely that the growth not only depends on the usual parameters (temperature, flux) leads to the risk that investigations in different experimental set-ups and/or at different times (e.g. with "aged" as compared to "new" substrates) can lead to controversial results, since the preparation parameters, e.g. the surface morphology, were not identical. In the present approach, i.e. using a multimethod spectro-microscope, such as the SMART, ${ }^{6-8,42,43}$ we could limit this risk since a combination of methods could be applied quasi simultaneously to the same sample, even to the same spot on the sample, in a single instrument. The methods used in the present work, i.e. UV-PEEM, NEXAFS-PEEM, LEEM, $\mu$-spot NEXAFS, and $\mu$-spot LEED, will be introduced together with the results in the following sections.

\section{Results and discussion}

The growth of atomic layers or organic films is a dynamic process, involving thermodynamic as well as kinetic aspects like adsorption, desorption, diffusion, nucleation, growth, agglomeration, etc. Usually, growth beyond the monolayer is studied by recording selected "snap shots", i.e. measurements for a static situation after preparation of a selected coverage. Dynamic processes like phase transitions, Oswald ripening, or influences of the substrate are then (indirectly) derived from comparisons of sequences of static results. However, some dynamic processes like the occurrence of meta-stable domains or the dynamical re-arrangement during the growth process can in many cases only be observed by a direct real-time investigation, i.e. by recording "movies" of the growth process.

\subsection{Direct observation of Stranski-Krastanov growth}

Fig. 1 shows micrographs from the layer growth of PTCDA on $\mathrm{Ag}(111)$ at two different substrate temperatures, $420 \mathrm{~K}$ (top row) and $375 \mathrm{~K}$ (bottom row), respectively. The images were selected from movies (see ESI, $\dagger$ Movie_1.mp4) with about three hundred of such frames representing the continuous growth between the bare surface and a coverage of $5 \mathrm{ML}$. In this case the two displayed sequences were recorded at different times from different samples. The applied method was UV-PEEM, and the recorded signal electron emission by UV-illumination.

Two different mechanisms contribute to the image contrast: work function change (here only in the range of the first layer) 
and attenuation. The former contrast arises because the energy of the UV photons $(h \nu=4.9 \mathrm{eV})$ is slightly larger than the work function of the clean $\mathrm{Ag}(111)$ surface $(4.74 \mathrm{eV})$ leading to a strong emission signal in the case of clean Ag due to electrons stemming from the Ag Fermi level. The adsorption of PTCDA on $\mathrm{Ag}(111)$ leads to an increase of the work function by about $0.1 \mathrm{eV}^{24}$ and hence to the formation of a higher potential barrier for substrate electrons. Since there is no occupied PTCDA level with sufficiently low binding energy, much less electrons from the substrate and no electrons from the PTCDA monolayer are emitted: the PTCDA covered areas appear dark in the UV-PEEM image.

For the second layer, the work function changes much less ( $\ll 0.1 \mathrm{eV}$ ), and higher layers do not influence the work function at all. ${ }^{24}$ Thus, the second contrast mechanism gains importance: attenuation. Even if the cut-off surface potential is (nearly) reached there is still some emission from the substrate because of the tails in the electron as well as in the photon distribution. These still lead to (minor) emission from the substrate which can be made visible by increasing the amplification of the electron detector (which is done in the image sequences of Fig. 1 beyond the monolayer). This emission is, however, further reduced by each additional layer due to attenuation by inelastic scattering of the emitted electrons by phonons and other electrons: the more adsorbed layers the darker the corresponding area in the image. The attenuation of the emitted electrons corresponds to an inelastic mean free path of less than $1 \mathrm{~nm}$, which is clearly different from a high value expected for very low kinetic energies in the so-called "universal curve". ${ }^{25,26}$

The deposition at $420 \mathrm{~K}$ (top row) indicates a classic StranskiKrastanov behaviour, in agreement with ex situ $\mathrm{AFM},{ }^{27}$ in situ SPA-LEED,$^{12}$ and grazing-incidence XRD-measurements. ${ }^{12}$ First a complete monolayer is formed (Fig. 1a), followed by a complete second layer (Fig. 1b), and thereafter a complete third layer (Fig. 1c). After completion of the third layer, a 3-dimensional island starts growing (Fig. 1c, dark spot at 4 o'clock position), which continues growing for higher coverages (Fig. 1d) while the coverage of the remaining surface stays constant (3 ML).

A few other observations are interesting to note: first, on the clean Ag(111) surface huge (monolayer) islands are formed with diameters of $10 \mu \mathrm{m}$ or more. This means that considerable diffusion of PTCDA molecules over large (many $\mu \mathrm{m}$ ) distances must take place on the clean $\mathrm{Ag}$ surface at this temperature. Second, the islands appear to be rather homogeneous with long $(\mu \mathrm{m})$ step edges which indicate a highly ordered adsorbate structure (see below). Thirdly, the situation changes somewhat if the surface is less smooth as seen in the Christmas-tree like substrate structure at the right hand side. Here the rim of this structure consists of step bunches, i.e. it is characterized by a high density of steps, while the large area on the left hand side is essentially smooth with only single atomic steps at large (a few $100 \mathrm{~nm}$ ) distances (see also below). We hence derive that large islands are formed on smooth surface areas with monoatomic steps while high step densities hinder the formation of large islands. This allows the conclusion that epitaxial growth over large distances is unlikely if the surface morphology is unsuited, for instance by the occurrence of a high step density. The latter usually strongly depends on the substrate material and the surface preparation. After deposition of one or two monolayers the diffusion barrier of step bunches for additional molecules is strongly reduced, which has been investigated as function of temperature. ${ }^{20}$

Fourthly, the situation slightly changes for the second layer. In this case, the islands are less homogeneous and have the tendency to become somewhat smaller (not seen in Fig. 1) in particular at lower temperatures (see below). This seems to indicate that the diffusion length and/or the direct lateral interaction within the islands is reduced, but there are no indications why that should be the case. Instead, we believe that a third effect, namely the lateral corrugation of the vertical potential plays the dominant role in the case of the monolayer, leading to a substrate-mediated, strongly attractive (lateral, intermolecular) interaction at the edge of monolayer islands. Fifthly, the islands of the first and the second layer (and the 3-dim islands) appear at different surface areas indicating that the nucleation of second and higher layers is not markedly influenced by the substrate surface. And sixthly, the 3-dim islands grow in lateral as well as in vertical direction. For instance, the island of Fig. 1d is at least about $60 \mathrm{ML}$ high (derived from the deposited amount and the geometric parameters).

The deposition at $375 \mathrm{~K}$ (lower row of Fig. 1) shows large differences. First, the islands, in particular those of the monolayer, are significantly smaller indicating a reduced diffusion length. This is not surprising in the light of the reduced substrate temperature. More surprising is certainly that the size of the islands (and hence the diffusion length) is still in range of $\mu \mathrm{m}$ although the adsorbate bonding in the monolayer is quite large. ${ }^{9,21}$ Second, the third layer is not completed before the fourth layer and even 3-dim islands start to grow (Fig. 1g). Thirdly, the fourth layer seems to be unstable because it disappears for higher coverages (Fig. 1h) at the expense of the 3-dim islands which are smaller but higher in number in this temperature range.

\subsection{Metastable layers}

This instability has been investigated in more detail as displayed in Fig. 2. In the upper row the first three images represent intermediate coverages of the series shown in the lower part of Fig. 1 whereas the fourth image is the same image as Fig. $1 \mathrm{~h}$ but with different magnification. It is clearly seen that at least four layers compete with each other (layers are indicated by numbers), and that islands of the fourth layer appear and disappear. It can also be observed that the 4th layer island at the right hand side indicated by a red oval in Fig. 2a first decreases (Fig. 2b) but then grows into the third dimension (Fig. 2c and d). This leads to the conclusion that the fourth layer is metastable even under growth conditions, i.e. under permanent flux of deposited molecules.

A closer inspection reveals that also small changes occur within the third layer. This is more clearly seen when the molecular flux is switched off (Fig. 2, lower row). The sequence of images represents the situation after shutter closure with the 


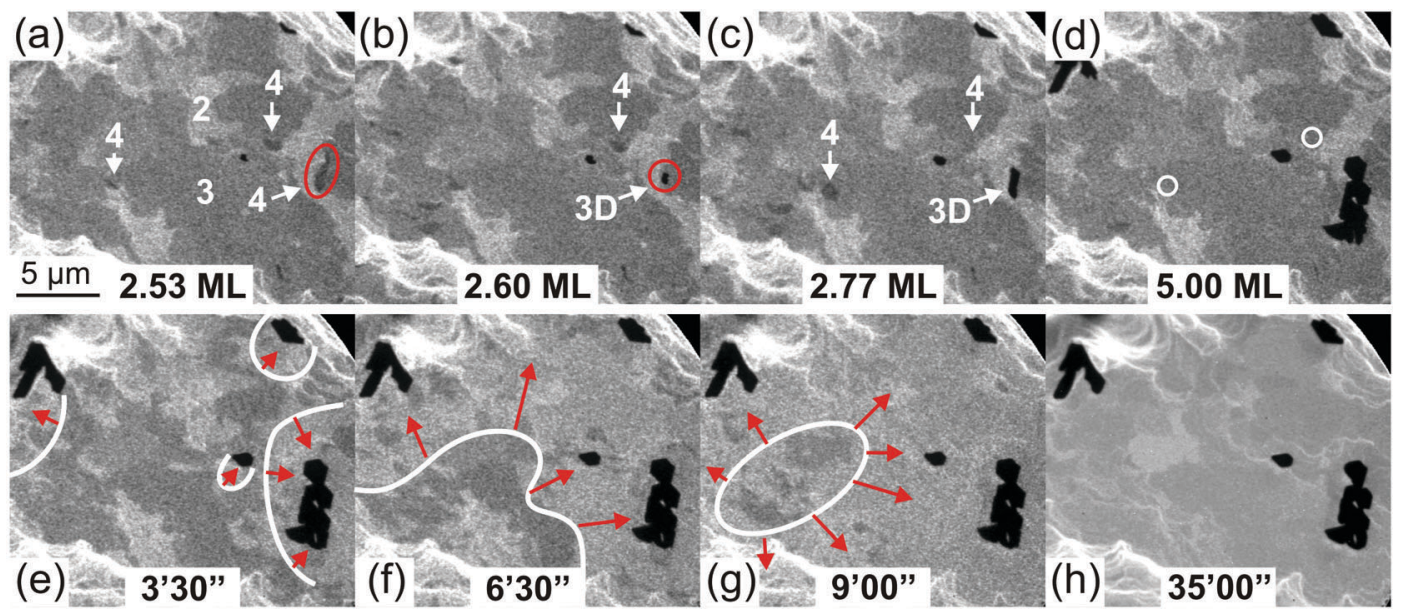

Fig. 2 Development of metastable layers: in the first row the images were taken during deposition at $375 \mathrm{~K}$ at the coverages indicated. In the second row the images were taken at the same temperature after deposition of $5 \mathrm{ML}$; the elapsed times indicated refer to the end of deposition.

elapsed times given in the figure. It is clearly seen that the third layer also disappears on the timescale of about $10 \mathrm{~min}$, and that it has completely disappeared after about half an hour (Fig. $2 \mathrm{~h}$ ). This means that also the third layer is metastable, and that desorption from and adsorption to the edges of the 3rd (and 4th) layer islands is in equilibrium under normal deposition conditions. By the way, in this case "desorption" does not mean desorption into the gas phase (vacuum) but dissolution from the 4th (3rd) layer island and diffusion to a 3-dim island via a two dimensional surface gas phase ${ }^{44}$ between them.

\subsection{Growth at lower temperatures, transition to Franck-van der Merwe behaviour}

The changes between the two temperatures of Fig. 1 call for a further lowering of the temperature in order to possibly achieve layer-by-layer growth. The result of two attempts is shown in Fig. 3, the selected temperatures being $355 \mathrm{~K}$ (upper row) and $320 \mathrm{~K}$ (lower row), respectively. At $355 \mathrm{~K}$, the trend of Fig. 1 is continued: smaller domains, higher density of islands, co-existence of several layers, 3rd and 4th layer metastable, competition of 3rd and 4th layer with 3-dim growth.

At $320 \mathrm{~K}$ we observe a nearly perfect layer-by-layer or Franckvan-der-Merwe growth. The first two layers grow successively (as before), for higher exposures several layers grow parallel. The detailed sequence (see ESI, $\dagger$ Movie_2.mp4) in fact reveals that the discernible large areas are filled stochastically, not all in parallel, but one after the other. This means, that, e.g., one domain with three layers may get a fourth, afterwards another with five layers a sixth, then another with four layers a fifth, then the same domain a sixth, and so forth. For all molecules or domains it is common, that the deposited molecules apparently diffuse over large distances (tens of $\mu \mathrm{m}$ ) before they get trapped at a rim of an island, and that the domains have the tendency to finish one layer before another domain (or the same) starts a new layer. Again the domain boundaries are determined by step bunches on the substrate surface, and the smooth areas are rather large (several $\mu \mathrm{m})$. By the way, in this case the half-moon shaped structures arose after an intense thermal treatment of the sample for cleaning purposes leading to a high rate of self-diffusion and the well-known formation of half-circle-shaped step bunches between pinning centres (defects). ${ }^{28-30}$

It should be emphasized at this point that the sequence shows true epitaxial multilayer growth of an organic layer on an inorganic substrate since (a) the organic domains are highly ordered as derived from (SPA-)LEED investigations (see ref. 13 and Section 3.5), (b) the molecular orientation is uniform within the entire film (see ref. 22 and Section 3.4), (c) the organic layer is in registry with the substrate, in this case even commensurate to the substrate (see ref. 13, 14, 16 and below), and (d) the single crystalline domains are rather large (several $\mu \mathrm{m}$ ) (see Section 3.5). The density of domain boundaries is in fact comparable to that of high quality epitaxial films of inorganic compound semiconductors.

The results of Fig. 4 were recorded at even lower temperatures, $270 \mathrm{~K}$ and $210 \mathrm{~K}$, respectively. In this case we switched to the LEEM mode of the SMART instrument, for several reasons: higher lateral resolution, higher intensity and therefore faster measurement, much less beam damage (as compared to X-ray irradiation), negligible heat transfer by irradiation, and demonstration of the suitability of LEEM (similar to PEEM) for the in situ observation of thin film growth. In the LEEM mode very low energy electrons (with kinetic energies at the surface of a few eV, here $1.4 \mathrm{eV}$ ) are back-diffracted from the surface and detected with high spatial resolution. ${ }^{31}$ The contrast arises (a) either because of the different crystal structure of the $\mathrm{Ag}$ substrate and the organic film and therefore different energy dependent reflectivity (dark Ag and brighter PTCDA layers) or (b) because of the interference of the partial electron waves reflected at the surface, at the organic planes and at the interface resulting in an energy- and thickness-dependent intensity variation and leading to contrast between different PTCDA film thicknesses. Note that the different contrast mechanism with respect to the PEEM mode (namely the interference condition) 

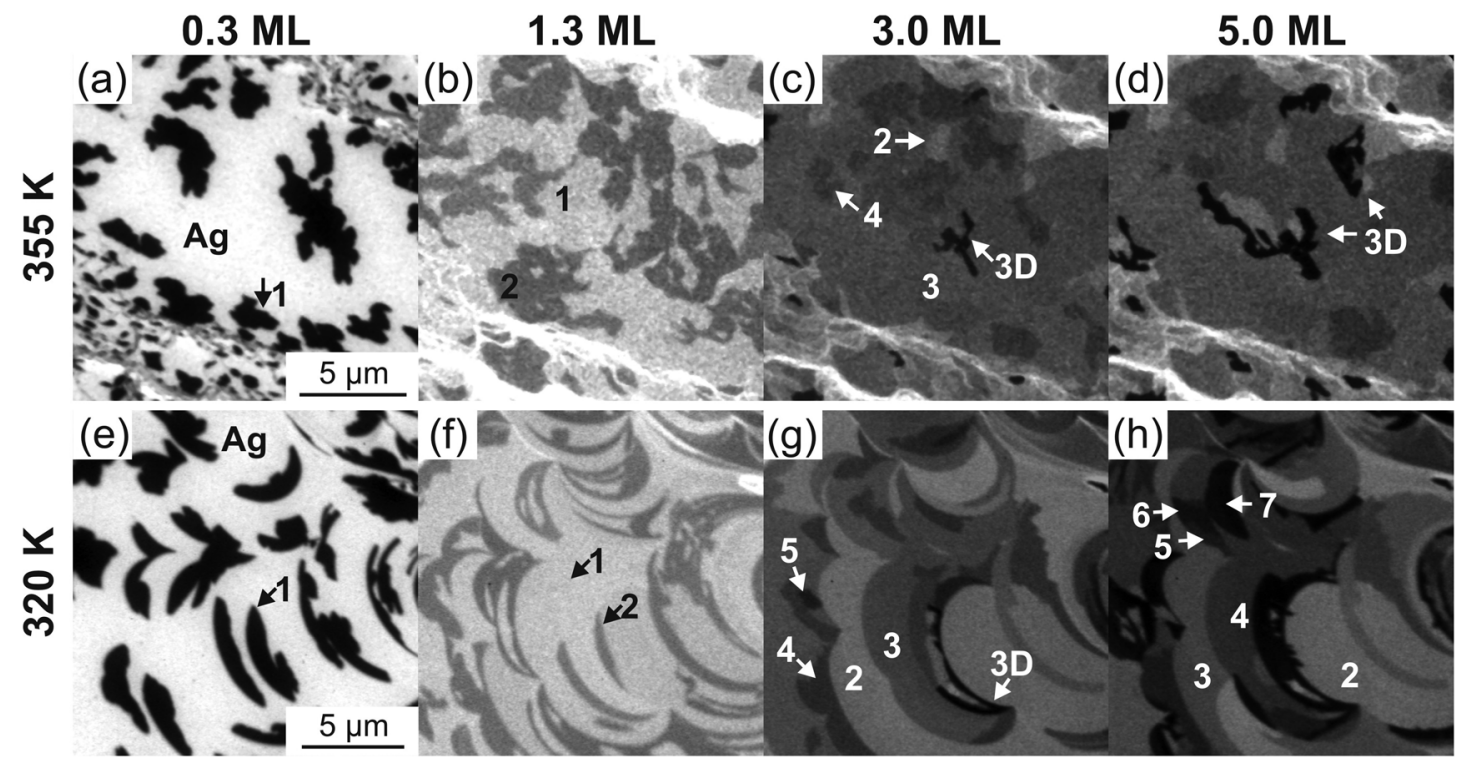

Fig. 3 Growth of PTCDA/Ag(111). UV-PEEM images taken during growth at 355 and $320 \mathrm{~K}$. The labels Ag, 1 to 7, and 3D are meant to help identifying the Ag substrate, the 1st to 7th PTCDA layer, and the three-dimensional islands. Since the intensities decrease exponentially with layer thickness, the differences between thicker layers (e.g. 7th $\mathrm{ML}$ ) and $3 \mathrm{~d}$-islands can hardly be seen in the images, but can easily be resolved by following the sequence of images (movie) of the film growth.

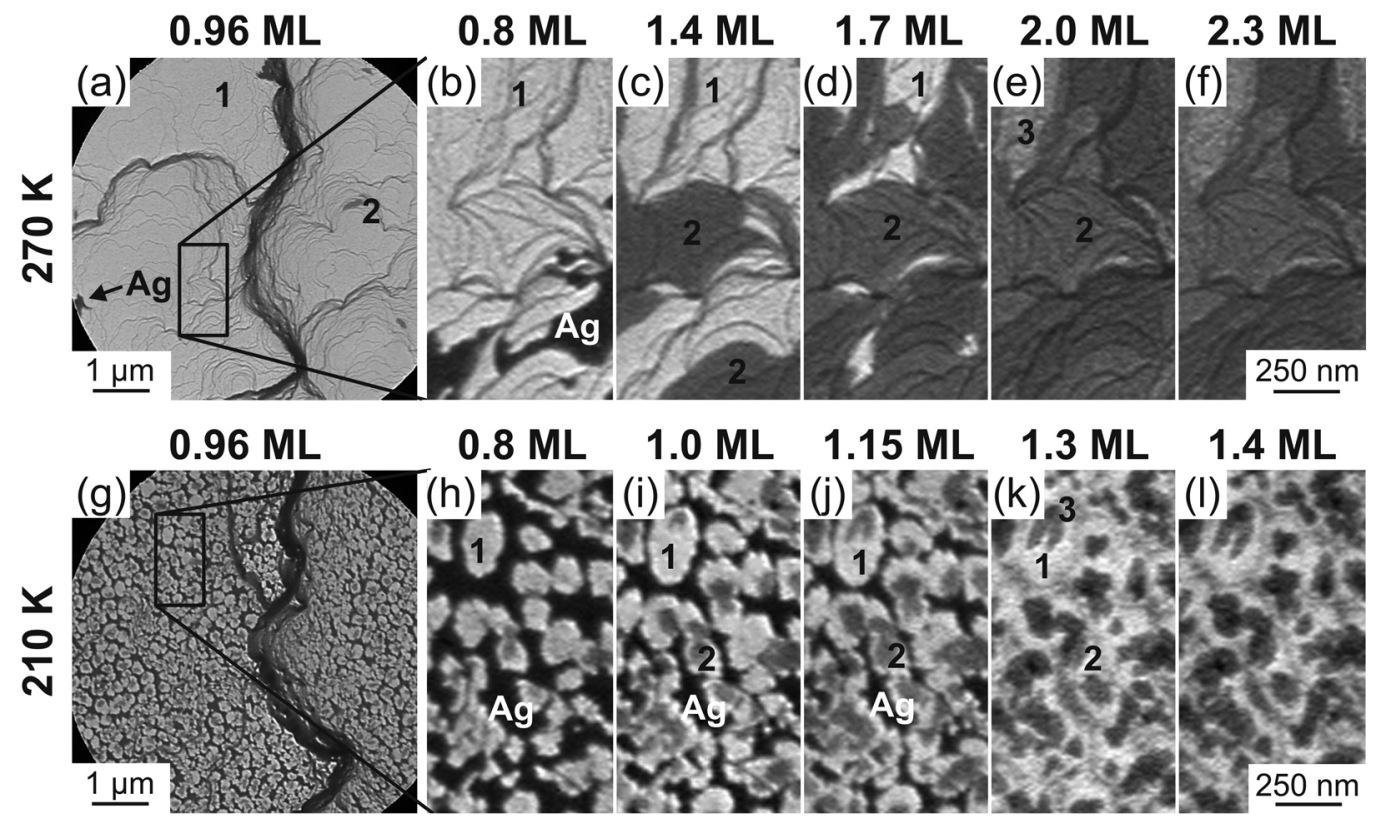

Fig. 4 LEEM series recorded with an electron energy of $1.4 \mathrm{eV}$; images show PTCDA growth behaviour at $210 \mathrm{~K}$ (bottom row) and $270 \mathrm{~K}$ (top row) on a clean $\mathrm{Ag}(111)$ surface. Note the nominal coverage of the $210 \mathrm{~K}$ series ranges only up to $1.4 \mathrm{ML}$ although the 3rd layer already started (e.g. seen at the terraces marked 1 and 3 ). In contrast, at $270 \mathrm{~K}$ the first two layers nearly fully close before the next starts. Note, that between frames (j) and ( $\mathrm{k}$ ) the focus of the instrument has been changed, such that dark areas now appear grey and the border lines are fuzzier.

causes a contrast reversal: PTCDA covered regions appear bright in the monolayer regime while the step bunches are darker, and under the present conditions - the second layer appears darker than the first, while the third layer is again brighter.

In the upper row of Fig. 4 (deposition at $270 \mathrm{~K}$ ) we observe similar initial growth behaviour as for $320 \mathrm{~K}$, i.e. layer-by-layer mode. In the experiments of this figure the higher spatial resolution allows a higher magnification. Whereas the first image has a similar field of view $(\sim 6 \mu \mathrm{m})$ as compared to Fig. 1-3 $(\sim 15 \mu \mathrm{m})$, the other four images display the close-up region indicated by the rectangle in the first image. The closeup has been chosen such that it contains flat areas separated by single atomic steps or bunches of few steps (thin and thick dark lines, respectively). One can clearly see that especially step 
bunches preferably act as boundaries for the formation of PTCDA domains, and that again PTCDA molecules diffuse over large distances to complete existing domains before new domains are nucleated. This is apparently true for the first three layers displayed in this figure but this behaviour most probably also continues for further layers. It is also worth mentioning that the "choice" of step bunches as domain boundaries changes from layer to layer which means that there are no particular bunches which act as nucleation centres or boundaries but that any step bunch can play this role. Moreover, one can again derive from this figure that the (atomic scale) morphology of the substrate surface plays a decisive role for the morphology of the growing organic film, at least for the first few layers.

Lowering the deposition temperature to $210 \mathrm{~K}$ changes the situation drastically as displayed in the lower sequence of images of Fig. 4. Now only relatively small islands $(50-300 \mathrm{~nm})$ are formed in the monolayer regime, and further deposition of molecules does not lead to a completion of the first layer but leads to the random growth of second and third layer patches as well. Note that in this case the coverage-dependent sequence of images stops already at $1.43 \mathrm{ML}$ because for higher coverages the situation becomes more and more fuzzy such that no additional information can be derived.

\subsection{Laterally resolved spectroscopic information from NEXAFS}

One of the most important properties of organic films is the orientation of the molecules. From integral NEXAFS (near edge $\mathrm{X}$-ray absorption fine structure) investigations on perfectly closed films (deposition at about room temperature) it is known that the planar PTCDA molecules in the film are oriented parallel to the substrate ("flat lying"). ${ }^{9,22,32}$ In the case of films with 3D islands (obtained at low, e.g. $<200 \mathrm{~K}$, or high substrate temperatures, e.g. $>400 \mathrm{~K})$ the interpretation of the integral data concerning the molecular orientation in the islands is less straightforward because the $3 \mathrm{D}$ islands cover only a few percent of the entire surface area and therefore hardly contribute to the integral NEXAFS signal. However, spectro-microscopy allows local probing and is therefore ideally suited to investigate inhomogeneous samples.

For the analysis of the molecular orientation the linear dichroism of NEXAFS can be used. ${ }^{33}$ This arises from the transition matrix element for the excitation of an electron from a core level into an unoccupied molecular orbital by absorption of an X-ray photon of the respective energy which is governed by selection rules. For PTCDA (and similar planar molecules) these selection rules lead to the result, that high NEXAFS intensity (bright intensity in Fig. 5) is observed when the linear polarization vector of the X-rays is oriented perpendicular to the molecular plane while the intensity is reduced to zero when the polarization vector is rotated parallel to the molecular plane with an angular dependence described by a $\sim \sin ^{2}$ function. ${ }^{22}$ Thus, in the experiment the angle between surface and light polarization has to be varied. Since in PEEM experiments this variation cannot be done by the common rotation of the sample (PEEMs require fixed sample geometry, i.e. surface normal

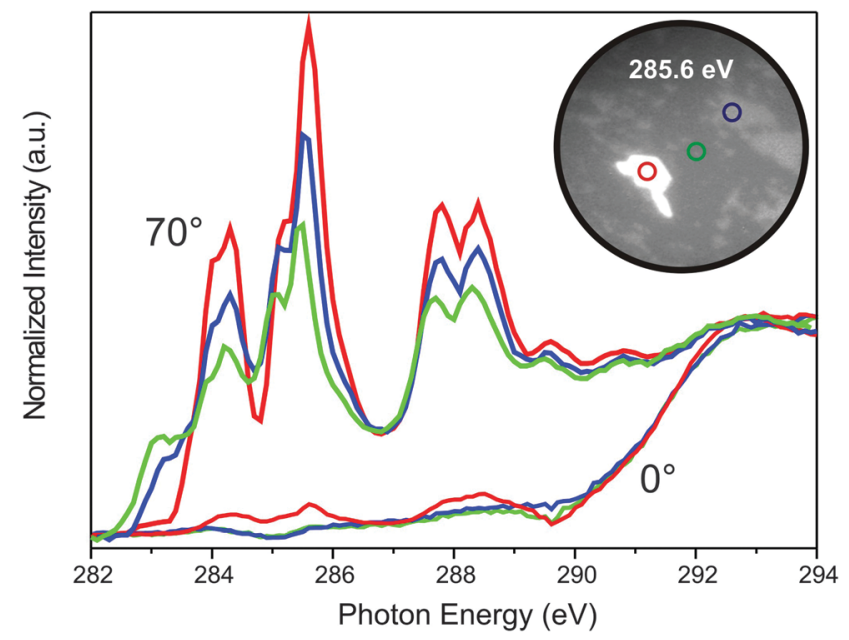

Fig. 5 Molecular orientation in NEXAFS. Comparison of the local NEXAFS spectra for two polarizations $\left(0^{\circ}\right.$ and $\left.70^{\circ}\right)$ and three sample regions (marked by coloured circles in the inset) for a PTCDA bi-layer (green), triple layer (blue) and 3D-island (red) on the Ag(111) surface.

along the optical axis of the objective lens) we used a different approach: the SMART is installed at a beamline with a so-called apple undulator, ${ }^{34,35}$ which enables the free choice of the orientation of the linear polarization (or helicity of circular polarization) of the light. In this way identical sample spots were imaged with two linear polarization orientations standing perpendicular to each other (using grazing incidence, i.e. $20^{\circ}$ ).

Fig. 5 shows an XPEEM image (enclosure) of a nominal $5 \mathrm{ML}$ thick PTCDA film as well as (coloured) NEXAFS spectra from three areas on the sample indicated by coloured circles in the enclosed image. The image taken at a photon energy of $285.6 \mathrm{eV}$ (recording secondary electrons) displays a sample area with one $3 \mathrm{D}$ island surrounded by areas covered with two and three layers of PTCDA, respectively. In this case the preparation is equivalent to that of Fig. 2d after cooling-down to RT for freezing the metastable third layer. While the image was recorded using upright $\left(70^{\circ}\right)$ polarization the NEXAFS spectra are displayed for both polarization directions: upright $\left(70^{\circ}\right.$, i.e. polarization nearly normal to the surface) and parallel (in-plane) polarization $\left(0^{\circ}\right)$. The $70^{\circ}$ spectra clearly show a set of $\pi$-resonances (excitations from $\mathrm{C}$ 1s orbitals into different unoccupied final states of $\pi$-symmetry) which have been interpreted in detail elsewhere. ${ }^{32}$

From the very large difference between the spectra taken with different polarizations one can unambiguously conclude that the PTCDA molecules are uniformly oriented with their molecular plane parallel to the surface with not much difference between bilayer, triple layer, and 3-dim island. At present it cannot be decided whether the very small $\pi$-signals from the 3-dim island for in-plane polarization $\left(0^{\circ}\right.$, red curve) are due to scattering effects, to a small tilt or bending of the entire island, or to single disordered molecules within the crystal. A particularly interesting result is that also molecules in the third, metastable layer is oriented parallel to the substrate (a significant tilt angle $>10^{\circ}$ can be excluded by a careful analysis of the intensity behaviour) since one may argue that metastable 
adsorbates may arise because of a less favourable orientation and bonding situation, respectively. Note that the information of a uniform parallel orientation is by no means trivial because other, very similar molecules, e.g. NTCDA with identical functional groups but with a smaller aromatic core, show a completely different behaviour and molecular orientation for coverages above a monolayer. ${ }^{36}$

Apart from the molecular orientation additional information is contained in the spectra. For the upright polarization one can distinguish spectroscopic differences between the spectra. First, the intensity of the $\pi$-transitions beyond $283.5 \mathrm{eV}$ is reduced for the bilayer as compared to that from the triplelayer and 3-d crystal. And secondly, an additional structure appears for the bilayer (and less for the triple-layer) spectra at $283 \mathrm{eV}$. The origin of these differences is the chemical bonding of the first layer to the $\mathrm{Ag}$ substrate which has been discussed in detail previously. ${ }^{9}$ Since the first layer considerably contributes to the bilayer and $\sim 50 \%$ less to the triple layer the signature gradually vanishes going from the mono- to multilayers. The spectroscopic signature can be qualitatively understood as chemisorptive (=covalent) bonding of the PTCDA molecules to the Ag substrate involving the frontier orbitals and leading to a mixing of $\mathrm{Ag} 5 \mathrm{~s}$ and $4 \mathrm{~d}$ states with $\pi$-orbitals of PTCDA. ${ }^{9}$ The new feature right at the Fermi level, i.e. the transition at $283 \mathrm{eV}$ represents the upper unoccupied part of a hybrid orbital which is partly occupied (and hence observable in photoemission) making the first molecular adsorbate layer "metallic".9,37 This example shows that also spatially-resolved chemical information can be obtained in the NEXAFS mode.

From spectra and images as those shown in Fig. 5 one can derive more quantitative information. We do this based on several sets of data ${ }^{38}$ but without showing these in detail here. For the preparation conditions of Fig. 5 (and Fig. 2d) we derive a coverage by 3 -dim islands of about $p=6.5 \%$ referred to the overall surface area. From this (and the calibrated exposure) we derive an average height of the 3-dim islands of about 48 layers (including the underlying $2 \mathrm{ML}$ ) while the total average coverage is $\theta_{\text {total }}=5 \mathrm{ML}$ corresponding to an average height of $15 \mathrm{~nm}$ (using a layer thickness of $0.325 \mathrm{~nm}$ ). This is in close agreement with the rough estimate of $\sim 60$ layers in 3-dim islands estimated above based on the UV-PEEM results for similar preparation conditions. Together with the information derived from UV-PEEM images that the average area of 3-dim islands is about $1.2 \mu \mathrm{m}^{2}$ we learn that the islands are rather flat objects with aspect ratios of roughly 60 for the preparation conditions given above.

\subsection{Laterally resolved structural information by reflected electrons}

After inspection of the initial growth behaviour discussed in context with Fig. 1-4 one would like to know more about the origin of the observed domains and their differences, in particular those of the first layer because they are the essential basis for further growth. Since with spectroscopic techniques no difference can be discerned, the domain structure may be due to structural differences. From detailed STM and LEED analyses it is known that the highly ordered monolayer is

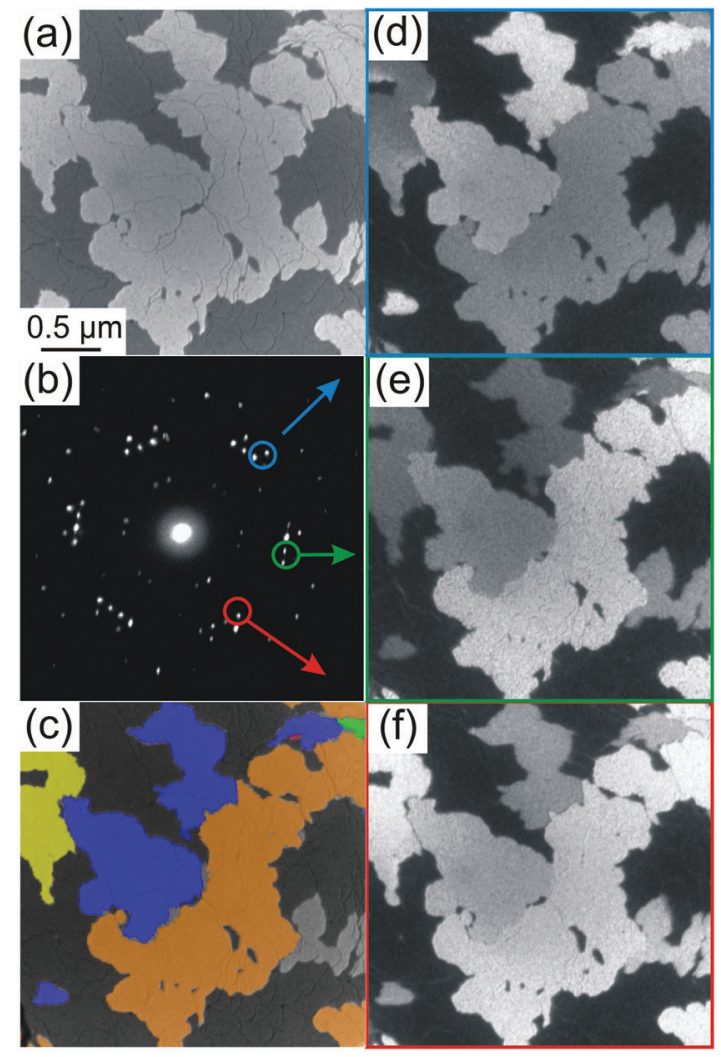

Fig. 6 Rotational domains in (sub-)monolayer PTCDA islands. The LEED pattern (b) from the LEEM image (a) consists of a superposition of 6 single domain patterns. Dark field images (right column, (d)-(f)) using significant LEED spots of (b) lead to the distinction of rotational domains. The arrangement of the domains is shown in the colour-labelled image (c).

commensurate to the $\operatorname{Ag}(111)$ surface and that six different azimuthal orientations occur due to the threefold rotational and twofold mirror symmetry. ${ }^{13-16}$

Fig. 6 summarizes LEED and LEEM data from about half a monolayer PTCDA on Ag(111) prepared similarly to the cases shown in Fig. 3a or e (substrate temperature: $340 \mathrm{~K}$ ). Fig. 6a displays a bright field LEEM image (using the specularly reflected (=back-diffracted) electrons; electron energy $2 \mathrm{eV}$ ) which is similar to those of Fig. 4. Like in Fig. 4b one can distinguish uncovered (dark) and covered (bright) areas which are subdivided in domains separated by substrate steps or step bunches. One observes that the islands also grow across substrate atomic steps (appearing as dark lines due to destructive interference). By switching the instrument setting from real space imaging to the diffraction mode one obtains the LEED pattern shown in Fig. $6 \mathrm{~b}$ (electron energy $4 \mathrm{eV}$ ). It shows the well-established LEED pattern of a highly ordered, commensurate PTCDA monolayer on $\mathrm{Ag}(111)$ which consists of six different domains and is understood in detail. ${ }^{13-16}$

Each spot of the LEED pattern (Fig. 6b) can be assigned to one of the six domains. By selecting one (or a few) of these spots by an aperture positioned in one of the diffraction planes of the SMART instrument the intensity in the LEEM image predominantly stems from that (or those) domains that belong to the 
selected superstructure spot(s). Such so-called dark field images are displayed on the right of Fig. 6(d)-(f). They stem according to the colour of the frames - from apertures that are set to either one of three positions indicated by the circles of corresponding colours in Fig. 6b. In the three dark field images the different domains display different brightness, in agreement with the basic idea sketched above. However, the intensity difference is clearly less than $100 \%$ because in each case the aperture accepted at least two superstructure spots as well as stray electrons from the background. However, the intensity difference is sufficient to find those domains that have the same intensity dependence and hence to assign each domain to one type of rotational domain. This is done in Fig. $6 \mathrm{c}$ which uses four different colours to identify different rotational domains.

Thus we learn from this figure that each area is delimited by steps or step bunches. Different nucleation centres are likely the origin of different rotational domains. The domains can propagate over (single or few) steps but are also visibly limited by step boundaries. As some step-enclosed areas do not lead to nucleation centres, we can derive that, at this temperature, PTCDA molecules ignore steps as minor obstacles and diffuse over macroscopic distances as testified by the rather large domains observed (several $\mu \mathrm{m}$ ). On the contrary, the variety of domain sizes observed suggests that bunches of several steps act as diffusion barriers, in agreement with a previous publication. ${ }^{20}$ In other words, the rotational domain sizes and hence the quality of the epitaxial layer crucially depend on the morphology of the substrate surface.

\section{Conclusions}

The present work gives comprehensive insight into the mechanisms and parameter dependences determining the epitaxial, or at least highly ordered, growth of an organic thin film on an inorganic substrate. The prototype system PTCDA on Ag(111) was chosen because many details are already known from other work which are complemented and completed by the present work thus yielding a coherent overall picture for one model system. In addition, the small lattice mismatch between the two rather different systems gives hope for true heterogeneous epitaxy which might be highly interesting for some applications. The latter aspect is nurtured by the finding that the optical properties strongly depend on the preparation conditions, for instance that the fluorescence yield can be about a factor of 10 larger if certain preparation conditions are chosen. ${ }^{4}$ The fact that some findings of the present paper have already been stated in previous publications using other methods is believed to be an advantage because it proves that the picture drawn here is consistent with previous work and that the combined data obtainable by the SMART can replace several investigations using a variety of different (complementary) techniques.

For the start of the growth process, i.e. for the nucleation of the first layer we here find the formation of very large, several micrometer-sized single-crystalline domains that grow in registry with the substrate surface structure (commensurate, i.e. epitaxial, adsorption) across surface steps or bunches of few steps (LEEM/ LEED results). This indicates huge diffusion lengths of PTCDA even on the clean $\mathrm{Ag}$ surface (tens of $\mu \mathrm{m}$ ) although the (final) bonding to the substrate is covalent (NEXAFS results) and site-specific (LEED results). Distinguishable domains of the first layer belong to the three rotational and two mirror domains and are usually separated by steps or multistep bunches. This new finding indicates a considerable influence of the morphology of the substrate surface on the domain structure of the organic film; hence the morphology of the substrate surface determines the "epitaxial quality", e.g. the density of domain boundaries in the growing molecular film (LEEM, UV-PEEM results). For temperatures above about $250 \mathrm{~K}$ we observe that the first layer is completed before the second layer starts growing, and the same happens then with the 2nd/3rd layer (UV-PEEM/LEEM results). In both layers the planar molecules are oriented parallel to the surface (NEXAFS results).

The growth of further layers strongly depends on the substrate temperature (consistently shown here) and deposition

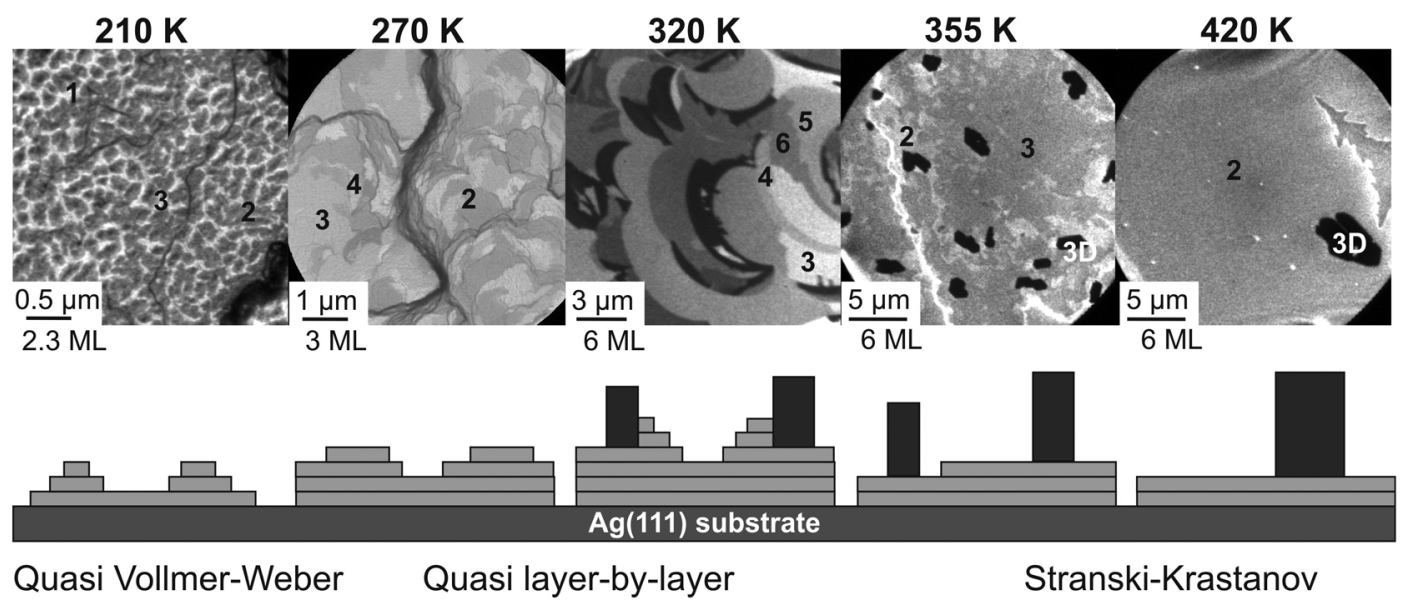

Fig. 7 Overview of the temperature dependant growth of the first 2 to 6 layers of PTCDA on Ag(111). Below RT, PTCDA starts in a quasi Vollmer-Weber mode, switches to Franck-van-der-Merwe mode around RT and transforms to a Stranski-Krastanov mode at elevated temperatures. Note the difference in lateral scales. Nominal coverage is denoted below the images. The $210 \mathrm{~K}$ and $270 \mathrm{~K}$ images were taken by LEEM, the others by UV-PEEM. 
rate (few data shown here). The temperature-dependent results are summarized in Fig. 7 which displays the most representative images for the temperatures discussed in this work (upper row) and the corresponding findings as schematic pictures. At the lowest temperature investigated here $(210 \mathrm{~K})$ we directly observed for the first time the nucleation of only small domains, the growth of up to three layers before the first is completed and hence the growth of small 3-dim islands which can be summarized as quasi Vollmer-Weber growth (from LEEM results).

At and above $270 \mathrm{~K}$ first two complete and stable PTCDA layers grow one after the other. Between 270 and $320 \mathrm{~K}$ this layer-by-layer growth is essentially continued such that one can consider this behaviour as Franck-van-der-Merwe growth mechanism which has been suggested before but never observed directly. It leads to a truly epitaxial film because the ordered geometric structure of the molecular film is in (commensurate) registry with the structure of the substrate (from PEEM and LEED results).

Above $320 \mathrm{~K}$ 3-dim islands start to grow after the second layer is completed leading to a clear Stranski-Krastanov layer growth behaviour. In the temperature range around $350 \mathrm{~K}$ we observe for the first time special growth conditions with metastable 3rd and 4th layers which are in equilibrium with the growing 3-dim islands. These metastable layers are present as long as the molecular flux is switched on but disappear with temperature-dependent lifetime as soon as the molecular flux is stopped (from PEEM results).

Finally we have to discuss the question why the observed change of the growth mechanism occurs. Stranski-Krastanov growth usually arises for thermodynamic reasons if a lattice mismatch between the structures of substrate and adlayer exists. By formation of 3-dim crystallites the contact area between the strained and the relaxed layer is reduced thus optimizing the total free energy.

In the present case this lattice mismatch between the $\mathrm{Ag}(111)$ surface and the (102) plane of crystalline $\beta$-PTCDA is about 1 to $2 \%$ while for $\alpha$-PTCDA (the most stable structure at RT) it is even larger $(5 \%){ }^{13}$ Thus, due to the strong substrate-PTCDA interaction at the interface the first PTCDA layer apparently grows in a strained fashion. ${ }^{13,39}$ Since also the second layer is slightly stronger bound to the first than to the third layer it also grows strained. Subsequent layers grow epitaxially (i.e. strained) if substrate temperature and deposition rate are low enough to prevent the thermodynamically favourable formation of 3-dim crystallites in the (relaxed) $\alpha$-structure which is probably hindered by activation barriers for nucleation and/or upward diffusion across the step edges of PTCDA layers (Ehrlich-Schwoebel barrier). The latter happens at elevated temperatures at which 3-dim crystallites on-top of the strained bi-layer are being formed. ${ }^{13}$ At intermediate temperatures (here around $350 \mathrm{~K}$ ) competition between the formation of (strained) 3rd and 4th layer and 3-dim growth leads to unstable conditions: 3rd and 4th layer are metastable and decay to 3-dim crystallites depending on temperature and time. We speculate that the metastability is most probably also existent for epitaxial multilayers ( $>4$ layers) at low temperatures, but the formation of crystallites is not observed within the timeframe of our experiment. Annealing experiments for (strained) low- $T$ layers have shown that at elevated temperatures (e.g. $400 \mathrm{~K})$ relaxation and formation of 3-dim crystals occur. ${ }^{13,39}$

It should be mentioned, that also an influence of the substrate morphology on the growth behaviour has been observed, e.g., on the nucleation site or on the nucleation density. ${ }^{20}$ In the present case, for instance, the 4th layer (appearing as dark grey area at $4 \mathrm{ML}$ in Fig. 2) nucleates only on flat surface areas, not at step bunches of the substrate (bright lines in the lower part of Fig. 2). This can be explained by the (additional) strain induced by steps and facets.

Summarizing the role of the specific instrument SMART for such investigations, we have benefited from the in situ combination of a variety of complementary surface techniques to address several surface properties ranging from microscopic insight, over topographic aspects, structural questions, electronic properties, chemical details up to dynamic processes. This enabled us to observe several new phenomena and to obtain a completely consistent picture. Moreover, all results could in principle be obtained from the same sample area or from the same microscopic object under identical preparation conditions using the spatially resolving techniques. Thus SMART is ideally suited to shed light on complicated microscopic properties and processes of surface systems.

\section{Acknowledgements}

This project was funded by the Federal German Ministry of Education and Research (BMBF) under contract 05 KS4WWB/4 and by the Fritz-Haber Institute of the Max-Planck Society. We thank the BESSY-II crew for technical support and the HZB for the allocation of synchrotron radiation beamtime.

\section{References}

1 B. Geffroy, P. le Roy and C. Prat, Organic Light-emitting Diode Technology: Materials, Devices and Display Technologies, Polym. Int., 2006, 55, 572.

2 C. Brabec, U. Scherf and V. Dyakonov, Organic Photovoltaics: Materials, Device Physics, and Manufacturing Technologies, John Wiley \& Sons, 2011.

3 M. Muccini, Nat. Mater., 2006, 5, 605.

4 M. Schneider and E. Umbach M. Sokolowski, Chem. Phys., 2006, 325, 185.

5 J. V. Barth, G. Costantini and K. Kern, Nature, 2005, 437, 671 .

6 R. Wichtendahl, R. Fink, H. Kuhlenbeck, D. Preikszas, H. Rose, R. Spehr, P. Hartel, W. Engel, R. Schlögl, H.-J. Freund, A. M. Bradshaw, G. Lilienkamp, T. Schmidt, E. Bauer, G. Benner and E. Umbach, Surf. Rev. Lett., 1998, 5, 1249.

7 R. Fink, M. R. Weiss, E. Umbach, D. Preikszas, H. Rose, R. Spehr, P. Hartel, W. Engel, R. Degenhardt, R. Wichtendahl, H. Kuhlenbeck, W. Erlebach, K. Ihmann, R. Schlögl, 
H.-J. Freund, A. M. Bradshaw, G. Lilienkamp, T. Schmidt, E. Bauer and G. Benner, J. Electron Spectrosc. Relat. Phenom., 1997, 84, 231.

8 T. Schmidt, U. Groh, R. Fink, E. Umbach, O. Schaff, W. Engel, B. Richter, H. Kuhlenbeck, R. Schlögl, H.-J. Freund, A. M. Bradshaw, D. Preikszas, P. Hartel, R. Spehr, H. Rose, G. Lilienkamp, E. Bauer and G. Benner, Surf. Rev. Lett., 2002, 9, 223.

9 Y. Zou, L. Kilian, A. Schöll, T. Schmidt, R. Fink and E. Umbach, Surf. Sci., 2006, 600, 1240.

10 F. S. Tautz, M. Eremtchenko, J. A. Schaefer, M. Sokolowski, V. Shklover, K. Glöckler and E. Umbach, Surf. Sci., 2002, 502/503, 176.

11 A. Hauschild, R. Temirov, S. Soubatch, O. Bauer, A. Schöll, B. C. C. Cowie, T.-L. Lee, F. S. Tautz and M. Sokolowski, Phys. Rev. B: Condens. Matter Mater. Phys., 2010, 81, 125432.

12 B. Krause, A. C. Dürr, K. Ritley, F. Schreiber, H. Dosch and D. Smiligies, Phys. Rev. B: Condens. Matter Mater. Phys., 2002, 66, 235404.

13 L. Kilian, E. Umbach and M. Sokolowski, Surf. Sci., 2004, 573, 359.

14 K. Glöckler, C. Seidel, A. Soukopp, M. Sokolowski, E. Umbach, M. Bohringer, R. Berndt and W.-D. Schneider, Surf. Sci., 1998, 405, 1.

15 F. S. Tautz, Prog. Surf. Sci., 2007, 82, 479.

16 E. Umbach, K. Glöckler and M. Sokolowski, Surf. Sci., 1998, 402-404, 20.

17 M. Eremtchenko, J. A. Schaefer and F. S. Tautz, Nature, 2003, 425, 602.

18 B. Stadtmüller, C. Henneke, S. Soubatch, F. S. Tautz and C. Kumpf, New J. Phys., 2015, 17, 023046.

19 T. Duden, A. Thust, C. Kumpf and F. S. Tautz, Microsc. Microanal., 2014, 20(03), 968-973.

20 H. Marchetto, U. Groh, T. Schmidt, R. Fink, H.-J. Freund and E. Umbach, Chem. Phys., 2006, 325, 178.

21 L. Kilian, A. Hauschild, R. Temirov, S. Soubatch, A. Schöll, A. Bendounan, F. Reinert, T.-L. Lee, F. S. Tautz, M. Sokolowski and E. Umbach, Phys. Rev. Lett., 2008, 100, 136103.

22 J. Taborski, P. Väterlein, H. Dietz, U. Zimmermann and E. Umbach, J. Electron Spectrosc. Relat. Phenom., 1995, 75, 129.

23 F.-J. Meyer zu Heringdorf, M. C. Reuter and R. M. Tromp, Nature, 2001, 412, 517-520.

24 This value has been determined by J. Taborski and E. Umbach using the method described in E. Umbach and R. Fink, in Proc. Intl. School Enrico Fermi, ed. G. La Rocca and V. Agranovich, North Holland, 2002, p. 233.

25 T. Graber, F. Forster, A. Schöll and F. Reinert, Surf. Sci., 2011, 606, 878.
26 M. B. Casu, Y. Zou, S. Kera, D. Batchelor, T. Schmidt and E. Umbach, Phys. Rev. B: Condens. Matter Mater. Phys., 2007, 76, 193311.

27 L. Chkoda, M. Schneider, V. Shklover, L. Killian, M. Sokolowski, C. Heske and E. Umbach, Chem. Phys. Lett., 2003, 371, 548.

28 M. Mundschau, E. Bauer and W. Telieps, Surf. Sci., 1989, 223, 413.

29 J. S. Ozcomert, W. W. Pai, N. C. Bartelt and J. E. Reutt-Robey, Surf. Sci., 1993, 293, 183.

30 G. A. Held, D. M. Goodstein, R. M. Feenstra, M. J. Ramstad, D. Y. Noh and R. J. Birgeneau, Phys. Rev. B: Condens. Matter Mater. Phys., 1993, 48, 8458.

31 E. Bauer, Rep. Prog. Phys., 1994, 57, 895.

32 A. Schöll, Y. Zou, D. Hübner, S. Urquhart, T. Schmidt, R. Fink and E. Umbach, J. Chem. Phys., 2005, 123, 044509.

33 J. Stöhr and D. A. Outka, Phys. Rev. B: Condens. Matter Mater. Phys., 1987, 36, 7891.

34 S. Sasaki, Nucl. Instrum. Methods Phys. Res., Sect. A, 1994, 347, 83.

35 J. Bahrdt, A. Gaupp, W. B. Peatman, F. Schaefers and C. Wang, Proc. SPIE, 1994, 168, 577.

36 U. Groh, Doctoral thesis, Universität Würzburg, Germany, 2006; U. Groh, T. Schmidt, H. Marchetto, E. Umbach, in preparation.

37 F. S. Tautz, M. Eremtchenko, J. A. Schaefer, M. Sokolowski, V. Shklover and E. Umbach, Phys. Rev. B: Condens. Matter Mater. Phys., 2002, 65, 125405.

38 H. Marchetto, Doctoral thesis, Freie Universität, Berlin, Germany, 2006; H. Marchetto, T. Schmidt, U. Groh, R. Fink, E. Umbach, in preparation.

39 B. Krause, A. C. Dürr, F. Schreiber, H. Dosch and O. H. Seeck, Surf. Sci., 2004, 572, 385.

40 D. Preikszas and H. Rose, J. Electron Microsc., 1997, 1, 1.

41 D. Preikszas, Doctoral thesis D 17, Technische Hochschule Darmstadt, Germany, 1995.

42 T. Schmidt, H. Marchetto, P. L. Levesque, U. Groh, F. Maier, D. Preikszas, P. Hartel, R. Spehr, G. Lilienkamp, W. Engel, R. Fink, E. Bauer, H. Rose, E. Umbach and H.-J. Freund, Ultramicroscopy, 2010, 110, 1358.

43 T. Schmidt, A. Sala, H. Marchetto, E. Umbach and H.-J. Freund, Ultramicroscopy, 2013, 126, 23.

44 A two dimensional gas describes a dilute layer of molecules that are weakly bound to the surface and diffuse freely across the surface. Hence, they are not directly imaged by most methods. See e.g. J. A. Venables, G. D. T. Spiller and M. Hanbücken, Rep. Prog. Phys., 1984, 47, 399 or E. Loginova, N. C. Bartelt, P. J. Feibelman and K. F. McCarty, New J. Phys, 2008, 10, 093026. 